\title{
A Stefan problem for a protocell model
}

\author{
Avner Friedman ${ }^{1}$ and Bei $\mathrm{Hu}^{2}$ \\ 1. Department of Mathematics, University of Minnesota, Minneapolis, MN 55455 \\ 2. Department of Mathematics, University of Notre Dame, Notre Dame, IN 46556
}

\begin{abstract}
The paper considers a simple model of radially symmetric cell which undergoes growth due to a continuous supply of nutrient, and disintegration as a result from the various tasks the cell performs. The boundary of the cell is a "free boundary", unknown in advance, which evolves by responding to both the growth and disintegration processes. If the nutrient concentration (at infinity) exceeds a certain critical number, then two stationary solutions exist. It is established, by rigorous mathematical proofs, that the stationary solution with the smaller radius is unstable, whereas the stationary solution with the larger radius is stable.
\end{abstract}

Keywords. protocell, parabolic system, free boundary problem.

\section{The model}

In this paper we consider a physico-chemical model of a self-maintaining protocell which undergoes process of growth and dissolution that mimics (but greatly simplifies) biological cells. The model was initiated and studied in [4] [5]. The protocell can be visualized as having a porous structure maintained by building materials with concentration $C$; the structure is sustained only as long as $C$ exceeds a critical concentration $C^{*}$. Metabolism is maintained by nutrient material with concentration $\sigma$ which is distributed in the entire space with $\sigma=\tau$ at $\infty(\tau>0)$. $C$ and $\sigma$ satisfy a coupled system of reaction diffusion equations:

$$
\begin{gathered}
c \frac{\partial C}{\partial t}-\Delta C=-\sigma, \quad \Delta \sigma+\sigma=0 \quad \text { in the cell, } \\
\Delta \sigma=0 \quad \text { outside the cell, }
\end{gathered}
$$

where $c$ is a positive constant. The constant $c$ is the quotient of the time scale of diffusion to the time scale of cell doubling. In cases of interest, such as in tumor growth [1], [2], $c$ is a very small constant.

On the boundary of the protocell $C=C^{*}$. The various tasks that the cell continuously performs take their toll on the cell: they cause it to shrink. This is modelled by disintegration at the boundary at a rate $\beta, \beta>0$. On the other hand the flux of building material at the boundary causes the cell to grow. The total result of these two effects is

$$
V_{n}=-\frac{\partial C}{\partial n}-\beta
$$

where $n$ is the exterior normal, and $V_{n}$ is the velocity of the boundary points in the direction $n$. 
We shall consider here only the case of a spherical cell (in 3-dimensions). Setting

$$
r=\sqrt{x_{1}^{2}+x_{2}^{2}+x_{3}^{2}}, \quad u=C^{*}-C
$$

and denoting the boundary of the cell by $r=s(t)$, we then have the following system for $u=u(r, t), \sigma=\sigma(r, t)$ and $r=s(t)$ :

$$
\begin{aligned}
& \left(\frac{\partial^{2}}{\partial r^{2}}+\frac{2}{r} \frac{\partial}{\partial r}\right) \sigma=\sigma \chi_{\{r<s(t)\}}(r, t) \quad \text { in } \mathbb{R}^{3}, \\
& \sigma \rightarrow \tau \quad \text { as }|x| \rightarrow \infty,
\end{aligned}
$$

and

$$
\begin{aligned}
& c u_{t}-\left(\frac{\partial^{2}}{\partial r^{2}}+\frac{2}{r} \frac{\partial}{\partial r}\right) u=\sigma \quad \text { if } r<s(t), t>0 \\
& u=0 \quad \text { on } r=s(t), t>0, \\
& u=u_{0}(r) \quad \text { for } t=0,
\end{aligned}
$$

and finally, the free boundary condition

$$
s^{\prime}(t)=-u_{r}(s(t), t)-\beta .
$$

As shown in [4], if $\tau / \beta$ is less than a critical number $\mu^{*}$ then no steady state solutions exist, whereas if $\tau / \beta$ is larger than $\mu^{*}$ then there exist two steady state solution, with free boundary radii $R_{0}^{-}$and $R_{0}^{+}, R_{0}^{-}<R_{0}^{+}$. Numerical results and some heuristic arguments are given in [4] to show that the solution with $R_{0}^{-}$is unstable whereas the solution with $R_{0}^{+}$is stable. The purpose of this paper is to give rigorous mathematical proofs of these results.

In $\S \S 2,3$ we establish various estimates and prove existence and uniqueness of the solution to $(1.1)-(1.6)$. In $\S 4$ we prove that the stationary solution corresponding to $R_{0}^{-}$is unstable. In $\S 5$ we prove that the stationary solution corresponding to $R_{0}^{+}$is stable if $c$ is suitably small.

\section{A priori bounds on the solution}

The function

$$
g(r)=\frac{r^{2}}{r-\tanh r} \quad(0<r<\infty)
$$

will play a fundamental role in what follows. One can easily verify that $r-\tanh r>0$, so that $g(r)>0$; furthermore, $g(r) \rightarrow \infty$ if $r \rightarrow 0$ or $r \rightarrow \infty$ and there is a unique $r=R^{*}$ where $g(r)$ attains its minimum $\mu^{*}$, i.e.,

$$
\min _{0<r<\infty} g(r)=g\left(R^{*}\right)=\mu^{*} .
$$

One can compute that

$$
R^{*} \approx 1.6061486, \quad \text { and } \quad \mu^{*} \approx 3.7739398 .
$$


The steady state solutions with free boundary $r=R_{0}$ are given by

$$
u(r)=\frac{\tau}{\cosh R_{0}}\left(\frac{\sinh R_{0}}{R_{0}}-\frac{\sinh r}{r}\right) \quad \text { for } r<R_{0},
$$

where $R_{0}$ satisfies

$$
\frac{\tau}{\beta}=\frac{R_{0}^{2}}{R_{0}-\tanh R_{0}}=g\left(R_{0}\right)
$$

If $\tau / \beta<\mu^{*}$ then there are no steady state solutions, whereas if $\tau / \beta>\mu^{*}$ then there are two solutions, $u_{R_{0}^{+}}$and $u_{R_{0}^{-}}$, corresponding to $R_{0}=R_{0}^{+}$and $R_{0}=R_{0}^{-}$, where $R_{0}^{-}<R^{*}<R_{0}^{+}$. As $\tau / \beta$ increases from $\mu^{*}$ to $\infty, R_{0}^{-}$decreases from $R^{*}$ to 0 and $R_{0}^{+}$increases from $R^{*}$ to $+\infty$.

For a given $s(t)$, equation (1.1) with the boundary condition (1.2) can be solved explicitly $([4](8),(9))$ :

$$
\sigma(r, t)= \begin{cases}\tau\left(1-\frac{s(t)-\tanh s(t)}{r}\right) & \text { for } r \geqslant s(t), \\ \tau \frac{1}{\cosh s(t)} \frac{\sinh r}{r} & \text { for } r<s(t) .\end{cases}
$$

Substituting (2.5) into (1.3), we get an equation for $u$ involving the free boundary:

$$
c u_{t}-\left(\frac{\partial^{2}}{\partial r^{2}}+\frac{2}{r} \frac{\partial}{\partial r}\right) u=\tau \frac{1}{\cosh s(t)} \frac{\sinh r}{r} \quad \text { if } r<s(t), t>0 .
$$

Further,

$$
\begin{aligned}
& u=0 \quad \text { on } r=s(t), t>0, \\
& s^{\prime}(t)=-u_{r}(s(t), t)-\beta \quad \text { if } t>0,
\end{aligned}
$$

and

$$
u=u_{0}(r) \quad \text { for } t=0 .
$$

Lemma 2.1 If a solution of (2.6)-(2.9) exists for the time interval [0,T], then

$$
\begin{gathered}
\frac{4 \pi}{3} s^{3}(t)=\frac{4 \pi}{3} s^{3}(0)+c \int_{\{r<s(0)\}} u_{0}(r) d V-c \int_{\{r<s(t)\}} u(r, t) d V \\
+4 \pi \tau \int_{0}^{t}\left[\frac{1}{g(s(\xi))}-\frac{\beta}{\tau}\right] s^{2}(\xi) d \xi
\end{gathered}
$$

for $0<t \leqslant T$.

Proof. By integration of (2.6) we get

$$
\begin{aligned}
c & \int_{\{r<s(t)\}} u(r, t) d V-c \int_{\{r<s(0)\}} u_{0}(r) d V \\
& =c \int_{0}^{t} \int_{\{r<s(\xi)\}} u_{t}(r, \xi) d V d \xi \\
& =\int_{0}^{t} \int_{\{r=s(\xi)\}} \frac{\partial u}{\partial r} d S+\tau \int_{0}^{t} \int_{0}^{s(\xi)} \frac{1}{\cosh s(t)} \frac{\sinh r}{r} 4 \pi r^{2} d r d \xi \\
& =4 \pi \int_{0}^{t}\left\{s^{2}(\xi)\left[-s^{\prime}(\xi)-\beta\right]+\tau[s(\xi)-\tanh s(\xi)]\right\} d \xi \\
& =\frac{4 \pi}{3}\left[s^{3}(0)-s^{3}(t)\right]+4 \pi \int_{0}^{t}\left\{\tau[s(\xi)-\tanh s(\xi)]-\beta s^{2}(\xi)\right\} d \xi,
\end{aligned}
$$


from which the lemma follows.

We shall henceforce assume that

$$
0 \leqslant u_{0}(r) \leqslant C_{0}(s(0)-r) \quad \text { for } 0 \leqslant r<s(0)
$$

where $C_{0}$ is a positive constant.

Theorem 2.2 If a solution $(u(r, t), s(t))$ of (2.6)-(2.9) exists for all $0<t<T(T \leqslant \infty)$, then

$$
s(t)<R \quad \forall 0<t<T
$$

where $R$ is a constant independent of $T$.

Proof. We distinguish two cases:

Case (i): $\tau / \beta \leqslant \mu^{*}$. Since $u_{0}(r) \geqslant 0$, we have, by the maximum principle, $u(r, t)>0$ for $0 \leqslant r<s(t)$. From (2.10) and the definition of $\mu^{*}$ we obtain

$$
\frac{4 \pi}{3} s^{3}(t) \leqslant \frac{4 \pi}{3} s^{3}(0)+c \int_{\{r<s(0)\}} u_{0}(r) d V-4 \pi \beta \int_{0}^{t}\left(1-\frac{1}{\mu^{*}} \frac{\tau}{\beta}\right) s^{2}(\xi) d \xi .
$$

It follows that

$$
s(t) \leqslant\left(s^{3}(0)+3 c \int_{0}^{s(0)} u_{0}(r) r^{2} d r\right)^{1 / 3} .
$$

Case (ii): $\tau / \beta>\mu^{*}$. In this case, the equation (2.3) has exactly two solutions $R_{0}^{-}$and $R_{0}^{+}$.

Setting

$$
C_{1}=\max \left(R_{0}^{+}, s(0)\right)
$$

we can choose (using (2.11)) a constant $R$ such that

$$
C_{1}^{3}+\frac{\tau}{2} c C_{1}^{3} R \leqslant R^{3}, \quad \text { and } 0 \leqslant u_{0}(r) \leqslant \frac{\tau}{2}(R-r), 0<r<s(0) .
$$

We shall prove that (2.12) holds for this choice of $R$. Indeed, if this is not true, then there is a first $t^{*}>0$ such that $s\left(t^{*}\right)=R$. Since $s(t)<R$ for $0<t<t^{*}$, we have, by comparison (using the maximum principle),

$$
0 \leqslant u(r, t) \leqslant \frac{\tau}{2}(R-r), \quad 0<r<s(t), 0<t \leqslant t^{*}
$$

Take $t_{1} \in\left[0, t^{*}\right)$ such that

$$
s\left(t_{1}\right)=C_{1}, \quad C_{1}<s(t)<R \quad \text { for } t_{1}<t<t^{*} .
$$

Then $\left\{\frac{\tau}{\beta}[s(\xi)-\tanh s(\xi)]-s^{2}(\xi)\right\} \leqslant 0$ for $t_{1}<t<t^{*}$ and $(2.10)$ yields

$$
\begin{aligned}
s^{3}(t) & <s^{3}\left(t_{1}\right)+3 c \int_{0}^{s\left(t_{1}\right)} u\left(r, t_{1}\right) r^{2} d r+3 \beta \int_{t_{1}}^{t}\left\{\frac{\tau}{\beta}[s(\xi)-\tanh s(\xi)]-s^{2}(\xi)\right\} d \xi \\
& \leqslant C_{1}^{3}+\frac{\tau}{2} c C_{1}^{3} R \leqslant R^{3} \quad \text { for } t_{1}<t \leqslant t^{*} .
\end{aligned}
$$


Thus $s\left(t^{*}\right)<R$, which is a contradiction.

From the uniform boundedness of $s(t)$ we can infer (by comparison) the uniform boundedness of $u(r, t)$ :

$$
0 \leqslant u(r, t) \leqslant C(R-r) \quad \text { where } R>\sup _{0 \leqslant t \leqslant T} s(t) .
$$

The next lemma gives a sharper estimate on $u(r, t)$ for $r$ near $s(t)$, as well as a useful estimate for $s^{\prime}(t)$.

Lemma 2.3 Let $R, C_{1}$ be positive constant for which

$$
\begin{gathered}
R>\sup _{0 \leqslant t \leqslant T} s(t), \\
0 \leqslant u_{0}(r) \leqslant C_{1} \tanh (c \beta s(0))\left\{1-e^{-c \beta(s(0)-r)}\right\} \quad \text { for } 0 \leqslant r<s(0) .
\end{gathered}
$$

Then there is a constant $K$ depending only on $C_{1}, R, \tau, c$ and $\beta$ such that

$$
0 \leqslant u(r, t) \leqslant K \tanh (c \beta s(t))\left\{1-e^{-c \beta(s(t)-r)}\right\} \quad \text { for } 0<t \leqslant T,
$$

and

$$
-\beta<s^{\prime}(t)<-\beta+c K \tanh (c \beta s(t)) \quad \text { for } 0<t \leqslant T
$$

Proof. By the maximum principle, $u(r, t)>0$ if $r<s(t)$ and $u_{r}(s(t), t)<0$; hence

$$
s^{\prime}(t)>-\beta \quad \text { for } t>0
$$

Let

$$
w(r, t)=K \tanh (c \beta s(t))\left\{1-e^{-c \beta(s(t)-r)}\right\},
$$

where we choose $K$ large enough so that

$$
w(r, 0) \geqslant u_{0}(r)
$$

Clearly,

$$
\begin{aligned}
c w_{t} & -\left(\frac{\partial^{2}}{\partial r^{2}}+\frac{2}{r} \frac{\partial}{\partial r}\right) w \\
& =K c \beta \tanh (c \beta s(t)) e^{-c \beta(s(t)-r)}\left\{c s^{\prime}(t)+c \beta+\frac{2}{r}+\frac{c\left[e^{c \beta(s(t)-r)}-1\right] s^{\prime}(t)}{\sinh (c \beta s(t)) \cosh (c \beta s(t))}\right\} \\
& \geqslant K c \beta \tanh (c \beta s(t)) e^{-c \beta(s(t)-r)}\left\{\frac{2}{r}-\frac{2 c \beta}{\cosh (c \beta s(t))}\right\} \\
& \geqslant(2-\sqrt{2}) K c \beta \tanh (c \beta s(t)) e^{-c \beta(s(t)-r)} \frac{1}{r}
\end{aligned}
$$

in deriving the above inequalities, we made use of (2.22) and of the inequalities:

$$
\begin{aligned}
& e^{\xi}-1 \leqslant 2 \sinh \xi \quad \text { for } \xi \geqslant 0, \\
& \cosh (\xi) \geqslant 1+\frac{\xi^{2}}{2} \geqslant \sqrt{2} \xi .
\end{aligned}
$$


We claim that for a suitable choice of the constant $K$,

$$
(2-\sqrt{2}) K c \beta \tanh (c \beta s(t)) e^{-c \beta(s(t)-r)} \frac{1}{r} \geqslant \tau \frac{1}{\cosh (s(t))} \frac{\sinh r}{r} \quad \text { for } 0<r<s(t)
$$

the proof of this inequality will be given later. If (2.24) holds then, by the maximum principle,

$$
u(r, t) \leqslant w(r, t)
$$

(note that $\left.w_{r}\right|_{r=0}<0,\left.u_{r}\right|_{r=0}=0$ so $w-u$ cannot take minimum at $r=0$ ). It follows that

$$
u_{r}(s(t), t) \geqslant w_{r}(s(t), t)=-K c \beta \tanh (c \beta s(t))
$$

and so

$$
s^{\prime}(t) \leqslant-\beta+K c \beta \tanh (c \beta s(t)) .
$$

To finish the proof of the lemma, it remains to verify (2.24). Observe that (2.24) is equivalent to

$$
(2-\sqrt{2}) K \tanh (c \beta s(t)) \geqslant \frac{\tau}{c \beta} \frac{e^{c \beta s(t)}}{\cosh s(t)} e^{-c \beta r} \sinh r \quad \text { for } 0<r<s(t) .
$$

The function $e^{-c \beta r} \sinh r$ is monotonically increasing in the following cases:

Case (i): $c \beta \leqslant 1$;

Case (ii): $c \beta>1, r \leqslant s(t) \leqslant \frac{1}{2} \log \frac{c \beta+1}{c \beta-1}$ for all $0 \leqslant t \leqslant T$.

In these two cases, it suffices to prove (2.27) for $r=s(t)$, so that, (2.27) holds if $K$ is such that

$$
(2-\sqrt{2}) K \geqslant \frac{\tau}{c \beta} \sup _{0<\xi<\infty} \frac{\tanh (\xi)}{\tanh (c \beta \xi)} \equiv \frac{\tau}{c \beta} \max \left(1, \frac{1}{c \beta}\right)
$$

Finally, if $c \beta>1$ and $\max _{0 \leqslant t \leqslant T} s(t)>\frac{1}{2} \log \frac{c \beta+1}{c \beta-1}$, we rewrite (2.24) in the form

$$
(2-\sqrt{2}) K \tanh (c \beta s(t)) e^{-(c \beta-1)(s(t)-r)} \geqslant \frac{\tau}{c \beta} \frac{e^{s(t)}}{\cosh s(t)} e^{-r} \sinh r \quad \text { for } 0<r<s(t)
$$

and observe that the function $e^{-r} \sinh r$ is monotonically increasing. It therefore suffices to prove (2.29) just for $r=s(t)$ on the right-hand side. But, since $s(t)<R$, this is a consequence of

$$
(2-\sqrt{2}) K e^{-(c \beta-1) R} \geqslant \frac{\tau}{c \beta} \sup _{0<\xi<\infty} \frac{\tanh (\xi)}{\tanh (c \beta \xi)} \equiv \frac{\tau}{c \beta}
$$

which holds if $K$ is chosen sufficiently large. 


\section{Existence and uniqueness}

In this section we assume, in addition to (2.11), that

$$
u_{0}^{\prime}(r) \text { is continuous if } 0 \leqslant r \leqslant s(0) .
$$

Theorem 3.1 Let (2.11) and (3.1) hold. Then

(i) There exists a unique solution $(u(r, t), s(t))$ of (2.6), (2.9) with $s^{\prime}(t)$ continuous for a time interval $0 \leqslant t<\delta_{0}$;

(ii) If a solution $(u(r, t), s(t))$ exists for $0 \leqslant t<T$ and $\liminf _{t \rightarrow T-0} s(t)>0$, then the solution can be continued to $0 \leqslant t \leqslant T+\delta$ for some $\delta>0$.

The proof, by a fixed point theorem for a contraction mapping, is similar to the corresponding proof for the Stefan problem [3, Chap. 8], and is therefore omitted.

Theorem 3.2 If

$$
\frac{\tau}{\beta}<\mu^{*},
$$

then there exists a finite $t^{*}$ such that the solution exists for $0 \leqslant t<t^{*}$ and $s(t) \rightarrow 0$ for $t \rightarrow t^{*}$, i.e., the cell shrinks to zero at time $t^{*}$.

Proof. From (2.13) we get

$$
s^{3}(t)+\delta \int_{0}^{t} s^{2}(\xi) d \xi \leqslant \widetilde{C}_{1}
$$

for some positive constants $\widetilde{C}_{1}$ and $\delta$.

By Theorem 3.1, the solution can be continued as long as $s(t)$ remains uniformly positive. If the assertion of the theorem is not true, then the solution exists (and $s(t)$ is positive) for all $0<t<\infty$. Let $K$ be as in Lemma 2.3 and take $s_{0}$ such that $K \tanh \left(\beta s_{0}\right)<\beta / 2$. Since $\int_{0}^{t} s^{2}(\xi) d \xi$ is uniformly bounded, there exists a $\widetilde{t}>0$ such that $s(\widetilde{t})<s_{0}$. By Lemma 2.3 and a continuation argument, we then have $s^{\prime}(t) \leqslant-\beta / 2$ for all $t>\widetilde{t}$. This is a contradiction to the assumption that $s(t)>0$ for all $t>0$.

In view of theorems 2.2 and 3.1, a solution $(u(r, t), s(t))$ exists for all $0 \leqslant t<\infty$ if and only if, for any $T>0$, there is $s>0$ such that

$$
\inf _{0 \leqslant t<T} s(t) \geqslant s>0 .
$$

Furthermore, by Lemma 2.3, the constant $s$ can be chosen to be independent of $T$ if a global solution exists.

Next we establish a lower bound for $s(t)$ in the case $\tau / \beta>\mu^{*}$.

Let $C_{1}, C_{2}$ and $D_{1}, D_{2}$ be positive constants such that

$$
\begin{aligned}
& C_{1} \geqslant R_{0}^{+}, \quad C_{1}^{3}+\frac{\tau}{2} c C_{1}^{3} C_{2}=C_{2}^{3}, \\
& D_{2} \geqslant R_{0}^{-}, \quad D_{1}=D_{2}\left(\frac{\tau}{2} c C_{2}+1\right)^{1 / 3} .
\end{aligned}
$$


Theorem 3.3 Assume that

$$
D_{1}<R_{0}^{+}
$$

If $s(0), u_{0}(r)$ satisfy

$$
\begin{aligned}
& D_{1} \leqslant s(0) \leqslant C_{1}, \\
& 0 \leqslant u_{0}(r) \leqslant \frac{\tau}{2}\left(C_{2}-r\right) \quad \text { for } 0<r<s(0),
\end{aligned}
$$

then there exists a global solution $(u(r, t), s(t))$ of (2.6)-(2.9) with

$$
s(t)>D_{2} \text { for all } t>0 .
$$

Proof. In view of (3.2), we can apply case (ii) of Theorem 2.2 to conclude that $s(t) \leqslant C_{2}$ and then, by the maximum principle,

$$
0 \leqslant u(r, t) \leqslant \frac{\tau}{2}\left(C_{2}-r\right) .
$$

It follows that if

$$
\begin{aligned}
& R_{0}^{-}<s(t)<R_{0}^{+} \quad \text { for } t_{1}<t<t_{2}, \\
& s\left(t_{1}\right)=\min \left[s(0), R_{0}^{+}\right], \quad s\left(t_{2}\right)=D_{2},
\end{aligned}
$$

then

$$
3 \int_{0}^{s\left(t_{2}\right)} u\left(r, t_{2}\right) r^{2} d r-3 \int_{0}^{s\left(t_{1}\right)} u\left(r, t_{1}\right) r^{2} d r \leqslant \frac{\tau}{2} C_{2} D_{2}^{3}=\frac{1}{c}\left(D_{1}^{3}-D_{2}^{3}\right)
$$

where the inequality follows from (3.8).

To prove the theorem it suffices to show that (3.7) holds as long as the solution exists. If this is not true then there is a smallest $t=t_{2}$ such that $s\left(t_{2}\right)=D_{2}$. By (3.4), (3.5) there exists a $t_{1}$ such that $t_{1}<t_{2}, s\left(t_{1}\right)=\min \left[s(0), R_{0}^{+}\right]$and $s\left(t_{2}\right)<s(t)<s\left(t_{1}\right)$ for $t_{1}<t<t_{2}$. As in case (ii) of Theorem 2.2,

$$
s^{3}\left(t_{1}\right)-D_{2}^{3}<3 c \int_{0}^{D_{2}} u\left(r, t_{2}\right) r^{2} d r-3 c \int_{0}^{s\left(t_{1}\right)} u\left(r, t_{1}\right) r^{2} d r
$$

and since $D_{1}^{3} \leqslant \min \left[s^{3}(0),\left(R_{0}^{+}\right)^{3}\right]$, this is a contradiction to (3.9).

Remark 3.1. If $c$ is suitably small, then (3.4) is satisfied. In this case we have, by (3.7), (3.8),

$$
D_{2}<s(t)<C_{2} \text { for all } t>0 \text {. }
$$

In particular, if

$$
R_{0}^{-}+2 \delta<s(0)<C_{1} \quad(\delta>0)
$$

and $c$ is sufficiently small, depending on $\delta$ and $C_{1}$, then

$$
R_{0}^{-}+\delta<s(t)<C_{1}+\delta, \quad \text { for all } t>0 .
$$




\section{$4 \quad R_{0}^{-}$is unstable}

From Lemma 2.3 we infer that if $s(0)$ is very small, then $s^{\prime}(t)$ remains uniformly negative as long as the solution exists. In this section we shall prove, under more general assumptions on $s(0)$, that $s(t)$ is monotone decreasing, and, in particular, deduce that the stationary solution corresponding to $R_{0}^{-}$is unstable.

\section{Theorem 4.1 Suppose that}

$$
\begin{aligned}
& u_{r}(r, 0) \geqslant-\frac{\tau}{\cosh s(0)} \frac{r \cosh r-\sinh r}{r^{2}}-\frac{\tau}{s(0)}\left[\frac{\beta}{\tau}-\frac{1}{g(s(0))}\right] r \\
& u_{r}(s(0), 0)>-\beta
\end{aligned}
$$

and

$$
s(0) \leqslant R_{0}^{-}
$$

Then $s^{\prime}(t)<0$ as long as $s(t)$ remains positive, and $s(t)$ shrinks to zero in finite time.

Proof. From (4.2) we deduce that $s^{\prime}(0)<0$ and, by continuity, $s^{\prime}(t)<0$ for small $t>0$. If $s^{\prime}(t)$ does not remain negative while $s(t)$ is positive, then there exists a first $t^{*}$ such that $s\left(t^{*}\right)>0, s^{\prime}\left(t^{*}\right)=0$. Since $s^{\prime}(t)<0$ for $0 \leqslant t<t^{*}$, we have

$$
\begin{aligned}
& u_{r}(s(t), t)>-\beta \quad \text { for } 0 \leqslant t<t^{*} \\
& 0<s(t)<s(0) \leqslant R_{0}^{-} \quad \text { for } 0 \leqslant t<t^{*}
\end{aligned}
$$

We introduce the auxiliary function

$$
w=u_{r}(r, t)+\frac{\tau}{\cosh s(t)} \frac{r \cosh r-\sinh r}{r^{2}}+\frac{\tau}{s(t)}\left[\frac{\beta}{\tau}-\frac{1}{g(s(t))}\right] r
$$

where $g(r)$ is defined in $(2.1)$.

Since $g^{\prime}(r)<0$ for $0<r \leqslant R_{0}^{-}, g\left(R_{0}^{-}\right)=\tau / \beta$ and $0<s(t) \leqslant R_{0}^{-}$, we have

$$
\left[\frac{\beta}{\tau}-\frac{1}{g(s(t))}\right]>0 \quad \text { for } 0<t<t^{*}
$$

Using the relation $(r \cosh r-\sinh r) / r^{2}=(\partial / \partial r)(\sinh r / r)$ we easily deduce that

$$
\begin{aligned}
-\Delta & \left(\frac{r \cosh r-\sinh r}{r^{2}}\right)+\frac{2}{r^{2}}\left(\frac{r \cosh r-\sinh r}{r^{2}}\right) \\
& =-\frac{\partial}{\partial r} \Delta \frac{\sinh r}{r}=-\frac{\partial}{\partial r} \frac{\sinh r}{r}=-\frac{r \cosh r-\sinh r}{r^{2}}
\end{aligned}
$$

Combining this relation with (4.4) and the inequality $s^{\prime}(t)<0$, we find that

$$
c w_{t}-w_{r r}-\frac{2}{r} w_{r}+\frac{2}{r^{2}} w>0 \quad \text { for } 0<t<t^{*}
$$


Clearly, $w(s(t), t)>0$ for $0<t<t^{*}$, and, by $(4.1), w(r, 0) \geqslant 0$. It follows, by the maximum principle, that $w(r, t)>0$ for $0<t<t^{*}$. Since $s^{\prime}\left(t^{*}\right)=0$, we also have (from the definition of $w$ and $g$ ) that $w\left(s\left(t^{*}\right), t^{*}\right)=0$ and, therefore, by the maximum principle,

$$
w_{r}\left(s\left(t^{*}\right), t^{*}\right)<0
$$

i.e.,

$$
u_{r r}\left(s\left(t^{*}\right), t^{*}\right) \leqslant-\frac{\tau}{s\left(t^{*}\right)}\left[\frac{\beta}{\tau}-\frac{3}{s\left(t^{*}\right)}+\frac{3 \tanh s\left(t^{*}\right)}{s^{2}\left(t^{*}\right)}+\tanh s\left(t^{*}\right)\right] .
$$

On the other hand from $s^{\prime}\left(t^{*}\right)=0$ we deduce that $u_{t}\left(s\left(t^{*}\right), t^{*}\right)=0$, so that

$$
\begin{aligned}
0=c u_{t}\left(s\left(t^{*}\right), t^{*}\right) & =u_{r r}\left(s\left(t^{*}\right), t^{*}\right)+\frac{2}{s\left(t^{*}\right)} u_{r}\left(s\left(t^{*}\right), t^{*}\right)+\frac{\tau}{\cosh s\left(t^{*}\right)} \frac{\sinh s\left(t^{*}\right)}{s\left(t^{*}\right)} \\
& \leqslant-\frac{\tau}{s\left(t^{*}\right)}\left[\frac{3 \beta}{\tau}-\frac{3}{s\left(t^{*}\right)}+\frac{3 \tanh s\left(t^{*}\right)}{s^{2}\left(t^{*}\right)}\right] \\
& =-\frac{3 \tau}{s\left(t^{*}\right)}\left[\frac{\beta}{\tau}-\frac{1}{g\left(s\left(t^{*}\right)\right)}\right]<0 \text { by }(4.4),
\end{aligned}
$$

which is a contradiction. Having now proved that $s^{\prime}(t)<0$ as long as $s(t)$ remains positive, we shall next show that $s(t)$ converges to zero in finite time. Indeed, otherwise the limit $s_{0}=\lim _{t \rightarrow \infty} s(t)$ lies in the interval $\left(0, R_{0}^{-}\right)$. By a standard theorem on parabolic equations [3, Chap. 6] $(u(r, t), s(t))$ converges to a stationary solution and, consequently, $s_{0}$ must coincide with either $R_{0}^{-}$or $R_{0}^{+}$, which is a contradiction.

If, in Theorem 4.1, we take $s(0)=R_{0}^{-}$, then we get:

Corollary 4.2 Suppose

$$
\begin{aligned}
& u_{r}(r, 0) \geqslant-\frac{\tau}{\cosh R_{0}^{-}} \frac{r \cosh r-\sinh r}{r^{2}} \quad \text { for } 0<r<R_{0}^{-}, \\
& s(0)=R_{0}^{-}, \quad u_{r}\left(R_{0}^{-}, 0\right)>-\beta .
\end{aligned}
$$

Then $s^{\prime}(t)<0$ as long as $s(t)$ remains positive, and $s(t)$ shrinks to zero in finite time.

Since there are arbitrarily small perturbations of the stationary solution

$$
\frac{\tau}{\cosh R_{0}^{-}}\left(\frac{\sinh R_{0}^{-}}{R_{0}^{-}}-\frac{\sinh r}{r}\right)
$$

which satisfy (4.5), (4.6), we conclude:

Corollary 4.3 The stationary solution (4.5) is unstable.

One can even choose small perturbations of the stationary solution with $s(0)>R_{0}^{-}$(but $s(0)-R_{0}^{-}$small) for which $s(t)$ shrinks to zero in finite time.

The method of proof of Theorem 4.1 can be extended to establish monotonic decrease of $s(t)$ under different assumptions on the data. We give here one example. 
Theorem 4.4 Suppose

$$
\begin{aligned}
& u_{r}(r, 0) \geqslant-\beta \frac{r}{s(0)}, \quad u_{r}(s(0), 0)>-\beta, \\
& \tau \leqslant \frac{3 \beta}{\tanh s(0)} .
\end{aligned}
$$

Then $s^{\prime}(t)<0$ as long as $s(t)$ remains positive.

The proof is essentially the same as the proof of Theorem 4.1, with $w$ replaced by $u_{r}(r, t)+$ $\beta r / s(t)$.

We note that Theorem 4.4 is not contained in Theorem 4.1 since, in general, $-\beta r / s(0)$ is not larger than the right-hand side of (4.1).

\section{$5 \quad R_{0}^{+}$is stable}

In this section we prove that the stationary solution corresponding to $R_{0}^{+}$is stable provided the coefficient $c$ is sufficiently small. As we note that in the Introduction in actual biological cells of interest, $c$ may indeed be very small. In the case $c=0$, the solution $(\varphi(r, t), R(t))$ of (2.6)-(2.9) can be computed explicitly [4]:

$$
\varphi(r, t)=\frac{\tau}{\cosh R(t)}\left(\frac{\sinh R(t)}{R(t)}-\frac{\sinh r}{r}\right) \quad \text { for } r<R(t)
$$

where the free boundary $R(t)$ satisfies:

$$
\frac{d R}{d t}=\tau \frac{R-\tanh R}{R^{2}}-\beta=\tau\left[\frac{1}{g(R)}-\frac{\beta}{\tau}\right] \equiv \tau\left(h(R)-\frac{\beta}{\tau}\right) .
$$

Note that

$$
\begin{array}{lll}
h(R)>\frac{\beta}{\tau} & \text { and } \quad \dot{R}>0 & \text { for } R_{0}^{-}<R<R_{0}^{+}, \\
h(R)<\frac{\beta}{\tau} & \text { and } \quad \dot{R}<0 & \text { for } R>R_{0}^{+},
\end{array}
$$

and $h^{\prime}\left(R_{0}^{+}\right)<0$. Hence, by standard ODE analysis it follows that, if $R(0)>R_{0}^{-}$, then

$$
\left|R(t)-R_{0}^{+}\right| \leqslant C e^{-\alpha t} \quad \forall t>0
$$

where $C, \alpha$ are positive constants.

In this section we want to extend this result to the case where $c$ is positive and small.

Theorem 5.1 Let $s(0)>R_{0}^{-}$. If $c$ is sufficiently small then the solution the solution $(u(r, t), s(t))$ of (2.6)-(2.9) exists for all $t>0$,

$$
\lim _{t \rightarrow \infty} s(t)=R_{0}^{+}
$$

and the convergence is exponentially fast. 
This establishes a global asymptotic stability of the stationary solution corresponding to $R_{0}^{+}$.

From Remark 3.1 we already know that the solution exists for all $t>0$ and that (3.11) holds, i.e.,

$$
R_{0}^{-}<\underline{S} \leqslant s(t) \leqslant \bar{S}<\infty \text { for all } t>0
$$

Lemma 5.2 Define

$$
\underline{R}=\liminf _{t \rightarrow \infty} s(t), \quad \bar{R}=\limsup _{t \rightarrow \infty} s(t) .
$$

If $c$ is sufficiently, then $\bar{R}=\underline{R}=R_{0}^{+}$.

Proof. By (5.4)

$$
R_{0}^{-}<\underline{R} \leqslant \bar{R} \leqslant \bar{S}
$$

We claim that

$$
R_{0}^{-}<\underline{R} \leqslant R_{0}^{+} \leqslant \bar{R} \leqslant \bar{S} .
$$

In fact, if $\underline{R}>R_{0}^{+}$, then $g(s(\xi))>\tau / \beta+\varepsilon$ for some small $\varepsilon>0$ and all sufficiently large $\xi$. Therefore,

$$
\int_{0}^{\infty}\left[\frac{1}{g(s(\xi))}-\frac{\beta}{\tau}\right] s^{2}(\xi) d \xi=-\infty,
$$

which is a contradiction to Lemma 2.1. This proves that $\underline{R} \leqslant R_{0}^{+}$. Similarly, $R_{0}^{-} \leqslant \bar{R}$.

Next, we claim that

$$
\begin{aligned}
& \liminf _{t \rightarrow \infty} u(r, t) \geqslant \frac{\tau}{\cosh \bar{R}}\left(\frac{\sinh \underline{R}}{\underline{R}}-\frac{\sinh r}{r}\right) \quad \text { uniformly for } r<s(t), \\
& \limsup _{t \rightarrow \infty} u(r, t) \leqslant \frac{\tau}{\cosh \underline{R}}\left(\frac{\sinh \bar{R}}{\bar{R}}-\frac{\sinh r}{r}\right) \quad \text { uniformly for } r<s(t) .
\end{aligned}
$$

To prove $(5.6)$, let $\varphi(r, t)$ be the solution of the following problem

$$
\begin{aligned}
& c \varphi_{t}-\Delta \varphi=\frac{\tau}{\cosh (\bar{R}+\varepsilon)} \frac{\sinh r}{r} \quad \text { for } r<\underline{R}-\varepsilon, t>T, \\
& \varphi(\underline{R}-\varepsilon, t)=0 \quad \text { for } t>T, \\
& \varphi(r, T)=0 \text { for } r<\underline{R}-\varepsilon,
\end{aligned}
$$

where $\varepsilon>0$ is small. If we take $T=T(\varepsilon)$ to be large enough, then

$$
\underline{R}-\varepsilon \leqslant s(t) \leqslant \bar{R}+\varepsilon \quad \text { for } t>T,
$$

and, by maximum principle, $u(r, t) \geqslant \varphi(r, t)$ for $t>T$. Hence

$$
\liminf _{t \rightarrow \infty} u(r, t) \geqslant \lim _{t \rightarrow \infty} \varphi(r, t)=\frac{\tau}{\cosh (\bar{R}+\varepsilon)}\left(\frac{\sinh (\underline{R}-\varepsilon)}{\underline{R}-\varepsilon}-\frac{\sinh r}{r}\right) .
$$

Letting $\varepsilon \rightarrow 0+$, we obtain the inequality (5.6). The inequality (5.7) can be established in a similar way. 
Next, we estimate $\bar{R}-R_{0}^{+}$in case $\bar{R}>R_{0}^{+}$. Choose a sequence $t_{j} \rightarrow \infty$ such that $s\left(t_{j}\right) \rightarrow \bar{R}$ and take $\widetilde{t}_{j}<t_{j}$ such that

$$
R_{0}^{+}+\frac{1}{j}=s\left(\widetilde{t_{j}}\right)<s(t) \quad \text { for } \widetilde{t}_{j}<t<t_{j} ;
$$

since $\liminf _{t \rightarrow \infty} s(t) \leqslant R_{0}^{+}<\bar{R}$, such a choice of $\widetilde{t}_{j}$ is possible. Then $g(s(\xi))>\tau / \beta$ for $\widetilde{t}_{j}<\xi<t_{j}$ and Lemma 2.1 implies that

$$
s^{3}\left(t_{j}\right)-s^{3}\left(\widetilde{t}_{j}\right) \leqslant 3 c \int_{0}^{s\left(\widetilde{t}_{j}\right)} u\left(r, \widetilde{t}_{j}\right) r^{2} d r-3 c \int_{0}^{s\left(t_{j}\right)} u\left(r, t_{j}\right) r^{2} d r .
$$

Using (5.6), (5.7) in the last inequality, and letting $j \rightarrow \infty$, we obtain

$$
\begin{aligned}
\bar{R}^{3}-\left(R_{0}^{+}\right)^{3} \leqslant & 3 c \int_{0}^{R_{0}^{+}} \frac{\tau}{\cosh \underline{R}}\left(\frac{\sinh \bar{R}}{\bar{R}}-\frac{\sinh r}{r}\right) r^{2} d r \\
& -3 c \int_{0}^{\underline{R}} \frac{\tau}{\cosh \bar{R}}\left(\frac{\sinh \underline{R}}{\underline{R}}-\frac{\sinh r}{r}\right) r^{2} d r \\
\leqslant & 3 c \tau \int_{0}^{R_{0}^{+}}\left(\frac{1}{\cosh \underline{R}} \frac{\sinh \bar{R}}{\bar{R}}-\frac{1}{\cosh \bar{R}} \frac{\sinh \underline{R}}{\underline{R}}\right) r^{2} d r \\
& +3 c \tau \int_{0}^{R_{0}^{+}}\left(\frac{1}{\cosh \bar{R}}-\frac{1}{\cosh \underline{R}}\right) r \sinh r d r .
\end{aligned}
$$

Since the last integrand is $\leqslant 0$, we obtain

$$
\begin{aligned}
\bar{R}^{3}-\left(R_{0}^{+}\right)^{3} & \leqslant c \tau\left(\frac{1}{\cosh \underline{R}} \frac{\sinh \bar{R}}{\bar{R}}-\frac{1}{\cosh \bar{R}} \frac{\sinh \underline{R}}{\underline{R}}\right)\left(R_{0}^{+}\right)^{3} \\
& =\frac{c \tau}{\cosh \underline{R} \cosh \bar{R}}[k(\bar{R})-k(\underline{R})]\left(R_{0}^{+}\right)^{3},
\end{aligned}
$$

where $k(r)=\sinh r \cosh r / r$; note that $k^{\prime}(r)>0$. Similarly, we have (with $s\left(\tau_{j}\right) \rightarrow \underline{R}, s\left(\widetilde{\tau}_{j}\right)=$ $R_{0}^{+}-1 / j, s(t)<R_{0}^{+}-1 / j$ if $\left.\widetilde{\tau}_{j}<t<\tau_{j}\right)$

$$
\underline{R}^{3}-\left(R_{0}^{+}\right)^{3} \geqslant-\frac{c \tau}{\cosh \underline{R} \cosh \bar{R}}[k(\bar{R})-k(\underline{R})]\left(R_{0}^{+}\right)^{3},
$$

provided $\underline{R}<R_{0}^{+}$. Notice that if $\bar{R}=R_{0}^{+}$(or $\underline{R}=R_{0}^{+}$) then (5.8) (or (5.9)) is trivially satisfied. Combining (5.8) with (5.9) we get

$$
\bar{R}-\underline{R} \leqslant \frac{c \tau}{A}(\bar{R}-\underline{R}),
$$

where

$$
A=\frac{\left(\bar{R}^{2}+\bar{R} \underline{R}+\underline{R}^{2}\right) \cosh \underline{R} \cosh \bar{R}}{2\left(R_{0}^{+}\right)^{3} k^{\prime}(\bar{R})} \geqslant A_{0},
$$

and $A_{0}$ is a positive constant depending only on $\underline{S}, \bar{S}$ and $R_{0}^{+}$. If $c \tau / A_{0}<1$, then $\bar{R}-\underline{R}=0$ and the lemma is proved.

Having proved (5.2), we shall next prove local stability of $R_{0}^{+}$with exponential convergence of $s(t)$. Introduce the functions

$$
\varphi(r, t)=\frac{\tau}{\cosh s(t)}\left(\frac{\sinh s(t)}{s(t)}-\frac{\sinh r}{r}\right),
$$


and $v=u-\varphi$. A direct computation shows that

$$
\begin{aligned}
& c v_{t}-\left(\frac{\partial^{2}}{\partial r^{2}}+\frac{2}{r} \frac{\partial}{\partial r}\right) v=-c \varphi_{t} \quad \text { for } 0 \leqslant r<s(t), \\
& v(s(t), t)=0, \\
& s^{\prime}(t)=-v_{r}(s(t), t)-\beta+\tau \frac{s(t)-\tanh s(t)}{s^{2}(t)} .
\end{aligned}
$$

Lemma 5.3 Suppose that $c \tau<3 / 2$. Then there exists an $\varepsilon>0$ such that if

$$
\left|s(0)-R_{0}^{+}\right|<\varepsilon^{2}, \quad\left|s^{\prime}(0)\right|<\varepsilon
$$

and

$$
\left|u_{0}(r)-\frac{\tau}{\cosh s(0)}\left(\frac{\sinh s(0)}{s(0)}-\frac{\sinh r}{r}\right)\right|<\frac{\tau}{7} \varepsilon\left(s(0)-\frac{r^{2}}{s(0)}\right),
$$

then a global solution $(u(r, t), s(t))$ exists and $s(t)$ converges to the steady state (corresponding to $R_{0}^{+}$) exponentially fast.

The proof requires the fact that $g^{\prime}\left(R_{0}^{+}\right)>0(g$ is defined in $(2.1))$, and thus does not apply to $R_{0}^{-}$(since $\left.g^{\prime}\left(R_{0}^{-}\right)<0\right)$.

Proof. Clearly,

$$
\begin{aligned}
\left|\varphi_{t}\right| & =\tau\left|s^{\prime}(t)\right|\left|\frac{s(t)-\tanh s(t)}{s^{2}(t)}-\frac{\sinh s(t)}{\cosh ^{2} s(t)}\left(\frac{\sinh s(t)}{s(t)}-\frac{\sinh r}{r}\right)\right| \\
& \leqslant \tau\left|s^{\prime}(t)\right| \max \left[\frac{s(t)-\tanh s(t)}{s^{2}(t)}, \frac{\sinh s(t)}{\cosh ^{2} s(t)}\left(\frac{\sinh s(t)}{s(t)}-\frac{\sinh r}{r}\right)\right] \\
& \leqslant \tau\left|s^{\prime}(t)\right| \max \left[\frac{s(t)-\tanh s(t)}{s^{2}(t)}, \frac{\sinh ^{2} s(t)}{s(t) \cosh ^{2} s(t)}\right] \\
& \leqslant \tau\left|s^{\prime}(t)\right| \frac{1}{s(t)} .
\end{aligned}
$$

We claim that

$$
\left|s^{\prime}(t)\right|<\varepsilon e^{-\varepsilon t} \quad \text { for } 0<t<\infty .
$$

By assumption (5.16) is satisfied for $t=0$. If the assertion (5.16) is not true, then there is a first $t^{*}$ such that $\left|s^{\prime}\left(t^{*}\right)\right|=\varepsilon e^{-\varepsilon t^{*}}$. Introduce the function

$$
w(r, t)=\frac{c \tau \varepsilon}{6-C^{*} \varepsilon} e^{-\varepsilon t}\left(s(t)-\frac{r^{2}}{s(t)}\right) \quad \text { for } 0 \leqslant t \leqslant t^{*},
$$

where $C^{*}>s^{2}(t)+2 c$ for all $0<t<\infty$. Then

$$
\begin{aligned}
c w_{t}-\Delta w & =\frac{c \tau \varepsilon}{6-C^{*} \varepsilon} \frac{1}{s(t)}\left[6+c\left(1+\frac{r^{2}}{s^{2}(t)}\right) s^{\prime}(t)-\varepsilon\left(s^{2}(t)-r^{2}\right)\right] e^{-\varepsilon t} \\
& \geqslant \frac{c \tau \varepsilon}{6-C^{*} \varepsilon} \frac{1}{s(t)}\left(6-C^{*} \varepsilon\right) e^{-\varepsilon t} \\
& \geqslant \frac{c \tau \varepsilon}{s(t)} e^{-\varepsilon t}
\end{aligned}
$$


and, by assumption, $w(r, 0)>|v(r, 0)|$. Recalling (5.15) and (5.12), (5.13), we can use the maximum principle to compare $\pm v$ with $w$ and conclude that

$$
|v(r, t)| \leqslant w(r, t) .
$$

Since $v(s(t), t)=w(s(t), t)$, we then also have

$$
\left|v_{r}(s(t), t)\right| \leqslant \frac{2 c \tau \varepsilon}{6-C^{*} \varepsilon} e^{-\varepsilon t} .
$$

Thus

$$
\left|s^{\prime}(t)+\beta-\tau \frac{s(t)-\tanh s(t)}{s^{2}(t)}\right| \leqslant \frac{2 c \tau \varepsilon}{6-C^{*} \varepsilon} e^{-\varepsilon t} .
$$

Let

$$
h(r)=\frac{1}{g(r)}, \quad f(t)=\tau \frac{h\left(R_{0}^{+}\right)-h(s(t))}{s(t)-R_{0}^{+}},
$$

where $g(r)$ is defined by (2.1). Then we can rewrite (5.17) in the form

$$
\left|\left(s(t)-R_{0}^{+}\right)^{\prime}+f(t)\left(s(t)-R_{0}^{+}\right)\right| \leqslant \frac{2 c \tau \varepsilon}{6-C^{*} \varepsilon} e^{-\varepsilon t} .
$$

It follows that

$$
\left|s(t)-R_{0}^{+}\right| \leqslant\left|s(0)-R_{0}^{+}\right| \exp \left(-\int_{0}^{t} f(\xi) d \xi\right)+\frac{2 c \tau \varepsilon}{6-C^{*} \varepsilon} \int_{0}^{t} \exp \left(-\varepsilon \eta-\int_{\eta}^{t} f(\xi) d \xi\right) d \eta .
$$

Notice that for $s(t)$ near $R_{0}^{+}, h\left(R_{0}^{+}\right)-h(s(t)) /\left(s(t)-R_{0}^{+}\right) \sim-h^{\prime}\left(R_{0}^{+}\right) \equiv \delta>0$. Thus, for $s(t)$ near $R_{0}^{+}, \tau\left(\delta+C^{*} \varepsilon\right) \geqslant f(t) \geqslant \tau\left(\delta-C^{*} \varepsilon\right)$. Consequently,

$$
\left|s(t)-R_{0}^{+}\right| \leqslant \frac{\varepsilon^{2}}{\tau\left(\delta-C^{*} \varepsilon\right)} e^{-\tau\left(\delta-C^{*} \varepsilon\right) t}+\frac{2 c \tau \varepsilon}{6-C^{*} \varepsilon} \frac{1}{\tau\left(\delta-C^{*} \varepsilon\right)-\varepsilon} e^{-\varepsilon t} .
$$

Using this in (5.18) we obtain

$$
\begin{aligned}
\left|s^{\prime}(t)\right| \leqslant & \frac{2 c \tau \varepsilon}{6-C^{*} \varepsilon} e^{-\varepsilon t}+|f(t)|\left|s(t)-R_{0}^{+}\right| \\
\leqslant & \frac{2 c \tau \varepsilon}{6-C^{*} \varepsilon} e^{-\varepsilon t} \\
& \quad+\tau\left(\delta+C^{*} \varepsilon\right)\left(\frac{\varepsilon^{2}}{\tau\left(\delta-C^{*} \varepsilon\right)} e^{-\tau\left(\delta-C^{*} \varepsilon\right) t}+\frac{2 c \tau \varepsilon}{6-C^{*} \varepsilon} \frac{1}{\tau\left(\delta-C^{*} \varepsilon\right)-\varepsilon} e^{-\varepsilon t}\right) \\
& <\varepsilon e^{-\varepsilon t} \quad \text { for } 0<t \leqslant t^{*},
\end{aligned}
$$

provided

$$
\frac{2 c \tau}{6-C^{*} \varepsilon}+\left[\frac{\tau\left(\delta+C^{*} \varepsilon\right) \varepsilon}{\tau\left(\delta-C^{*} \varepsilon\right)}+\frac{2 c \tau}{6-C^{*} \varepsilon} \frac{\tau\left(\delta+C^{*} \varepsilon\right)}{\tau\left(\delta-C^{*} \varepsilon\right)-\varepsilon}\right]<1,
$$

which is satisfied if $c \tau<3 / 2$ and $\varepsilon$ is sufficiently small, and this is a contradiction to the assumption that $\left|s^{\prime}\left(t^{*}\right)\right|$ is equal to $e^{-\varepsilon t^{*}}$. Finally, from (5.19), we deduce that $s(t)$ converges to $R_{0}^{+}$exponentially fast.

Proof of Theorem 5.1. From Lemma 5.2 and (5.6), (5.7) we see that the assumptions of Lemma 5.3 are satisfied at some sufficiently large time $t=T$. It follows that

$$
\begin{aligned}
& \left|s(t)-R_{0}^{+}\right| \leqslant C e^{-\alpha t}, \\
& \left|s^{\prime}(t)\right| \leqslant C e^{-\alpha t}
\end{aligned}
$$


for all large enough $t$, where $C, \alpha$ are positive constants, and this completes the proof of Theorem 5.1.

Remark 5.1. The above proof shows that if (3.6) and (3.10) hold then the assertion of Theorem 5.1 holds for any $0<c<c^{*}$ where $c^{*}$ depends only on $\delta, C_{1}$ and $C_{2}$, in addition to $\tau$ and $\beta$.

Acknowledgment. The first author is partially supported by National Science Foundation Grant DMS \#9703842. The second author is grateful for the partial support from the Institute for Mathematics and its Application during his visit there.

\section{References}

[1] H.M. Byrne And M.A.J. Chaplain, Growth of nonnecrotic tumors in the presence of inhibitors, Math. Biosciences, 130, 151-181, 1995.

[2] H.M. Byrne And M.A.J. Chaplain, Growth of nonnecrotic tumors in the presence of inhibitors, Math. Biosciences, 135, 187-216, 1996.

[3] A. Friedman, Partial Differential Equations of Parabolic Type, Prentice-Hall, Englewood Cliffs, N. J. 1964.

[4] H. Schwegler, K. Tarumi and B. Gerstmann, Physico-chemical model of protocell, J. Math. Biology, 22, 335-348, 1985.

[5] K. Tarumi and H. Schwegler, A nonlinear treatment of the protocell by boundary layer approximation, Bull. Math. Biology, 49, 307-320, 1987. 